\title{
User Verification by Combining Speech and Face Biometrics in Video
}

\author{
Imran Naseem ${ }^{1}$ and Ajmal Mian ${ }^{2}$ \\ 1 School of Electrical, Electronic and Computer Engineering \\ naseei01@student.uwa.edu.au \\ 2 School of Computer Science and Software Engineering \\ The University of Western Australia \\ ajmal@csse.uwa.edu. au
}

\begin{abstract}
In this paper, physiological biometrics from face are combined with behavioral biometrics from speech in video to achieve robust user authentication. The choice of biometrics is motivated by user convenience and robustness to forgery as it is hard to simultaneously forge these two biometrics. We used the Mel Frequency Cepstral Coefficients for text-independent speaker recognition and local scale invariant features for video-based face recognition. Results of the two classifiers were fused using a weighted sum rule and an equal error rate of $0.6 \%$ was achieved on the VidTIMIT audio-visual database. We also performed identification experiments and achieved a combined identification rate of $99.13 \%$ on the same database.
\end{abstract}

\section{Introduction}

Access control has become a critical requirement in the modern world. The aim of access control is to allow only authorized personnel enter restricted areas (e.g military installations) or use sensitive resources (e.g. bank accounts). Differentiating between authorized users and impostors requires some sort of authentication which is defined as the process of verifying an individual's claimed identity. There are four possible means of authentication: (1) Something that we know (e.g. passwords, PIN); (2) Something that we have (e.g. ID cards, passports). (3) Something that we are (e.g. face, fingerprints); and (4) Something that we produce (e.g. gait, voice);

The first two means are non-biometric and hence not tied to the identity i.e. they can be forgotten, lost or stolen. Authentication based on password-only is vulnerable to eavesdropping and dictionary attacks. People forget their passwords, choose easy passwords which can be guessed, write their passwords and others read them. Combining passwords with the second means (i.e. ID cards) increases the robustness of access control but at the cost of more inconvenience to the users. An average person usually has more than one bank account, a credit card, an office access card and some membership cards (e.g. library, gymnasium). Carrying these cards and remembering a password (or PIN) for each of them becomes quite difficult. 
The last two means of authentication are called physiological and behavioral biometrics respectively. Physiological biometrics are based on the direct measurement of a part of the human body ("something we are"). Behavioral biometrics are based on the measurements of the actions taken by an individual ("something we produce"). Biometric identification is a one-to-many matching process whereby an identity is associated with an individual by matching his/her biometrics with the template of every identity in the database. Biometric authentication (or verification), is a one-to-one matching process whereby an individuals biometrics are matched with only the template of the claimed identity in order to allow or deny access. In this paper, the term "recognition" is also used to refer to identification.

Biometrics are naturally tied to their respective identities and hence cannot be lost, stolen or forgotten. Moreover, it is comparatively difficult to forge biometrics. Especially, behavioral biometrics are the hardest to be forged. In this paper, we combine physiological biometrics (face) and behavioral biometrics (speech) in video to achieve robust user authentication. The proposed algorithm is fully automatic including the offline training phase.

\section{$2 \quad$ Literature Review}

Among the behavioral biometrics, speech is perhaps the most convenient choice. Automatic speaker recognition (ASR) systems identify people utilizing the utterances. Depending upon the nature of the application, speaker identification or speaker verification systems, could be modeled to operate either in textdependent or text-independent modes. For text-dependent ASR, the user is required to utter a specific password, while for text-independent ASR, there is no need for such a constraint. Success in both cases depends on the modeling of speech characteristics which distinguish one user from the other. Text-dependent ASR is used for applications where the user is willing to cooperate by memorizing the phrase or password to be spoken which could be inconvenient to some users. Therefore, in this paper, our focus is on text-independent ASR which is considered to be a more challenging problem [1].

Research in the field of speaker recognition traces back to the early 1960s when Lawrence Kersta at Bell Labs [2] made the first major step in speaker verification by computers. He proposed the term voiceprint for a spectrogram, which was generated by a complicated electro-mechanical device [2]. Since then, there has been a tremendous amount of research in the area. Starting from spectrogram comparisons [2], passing through simple template matching [1], dynamictime wrapping [1], to more sophisticated statistical approaches like Gaussian Mixture Model (GMM) [3,4], Hidden Markov Model (HMM) [5-7] and neural networks [8,9]. Mel Frequency Cepstral Coefficients or MFCC features [2] have also been used for text-independent speaker recognition.

Face recognition research has also been around for over three decades with comprehensive surveys like Zhao et al. [10]. Classical face recognition research was based on matching single pairs holistic facial images [11]. Later, multiple independent images per individual were used to train a Linear Discriminant 
Analysis (LDA) classifier and recognition was performed on a single test image [12]. These techniques did not cope well with changes in illumination, pose and facial expressions e.g. while speaking. Video-based face recognition has become popular over the past few years because of the common availability of video cameras and their ability to capture more information. Moreover, motion helps in the recognition of faces [10]. Early video-based face recognition algorithms were frame based however, recent techniques match video sequences and use temporal coherence between the query and database videos in addition to the spatial information contained in individual frames [13]. Recognition systems can be trained offline once only [13], or even online [14]. Hybrid training approaches learn generic or specific face models offline in a batch mode and continuously updates the models during online recognition [15].

Most existing video face recognition algorithms assume a prior knowledge of the pose and pre-defined facial landmarks [16]. Some, rely on supervised learning where each frame is manually assigned to a cluster [15]. Manual clustering could be very laborious and time consuming due to the bulk of video data. Video-based face recognition research has mainly focused on the holistic face [10]. However, holistic or global features are sensitive to registration errors, occlusions, and variations in pose, illumination and facial expressions. Local features, on the other hand, do not require perfect normalization of the faces before matching and are therefore, more robust. Sivic et al. [17] used local features for retrieving different shots of a person from a movie. They extracted local features from pre-defined landmarks on the face and therefore, the accuracy of landmark identification is critical in their algorithm. A downside of local features is that rather than matching a single global feature per face, more local features are required to be matched resulting in added computational complexity. However, this is not a problem in verification which is a one-to-one matching process.

Multi-modal biometric research has recently gained popularity. Biometrics from independent modalities complement each other and increase the accuracy and robustness of the system. Speech and face are natural choices for multimodal biometric applications because they can be simultaneously acquired with video cameras. A review of audio-visual person identification and verification is given by Sanderson and Paliwal [18]. A detailed book on the subject including fusion techniques is also available [19].

\section{Speech Independent Speaker Recognition}

In this paper, we use the MFCC features for text-independent speaker recognition. MFCC is a popular feature extraction technique for speech signals [2]. The main idea behind MFCC features is to imitate the behavior of a human ear. Psychophysical studies have shown that human perception of the frequency contents of sounds for speech signals does not follow a linear scale [20]. Thus for each tone with an actual frequency $f$, measured in Hertz, a subjective pitch is measured on a scale called the $\mathrm{Mel}$ Scale $[21,22,20]$. The Mel-frequency scale has linear frequency spacing below $1000 \mathrm{~Hz}$ and a logarithmic spacing above 1 $\mathrm{KHz}$ as shown in Fig. 1-a. As a reference point, the pitch of a $1 \mathrm{KHz}$ tone, 40 


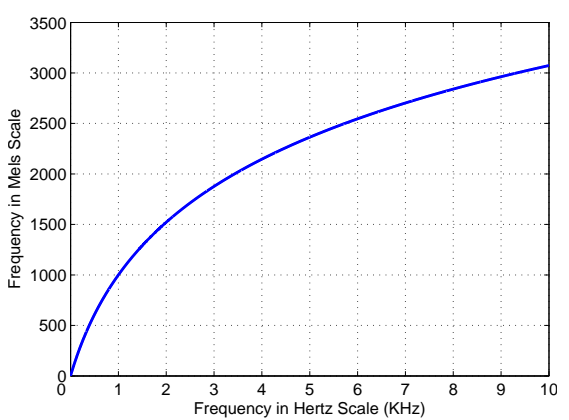

(a)

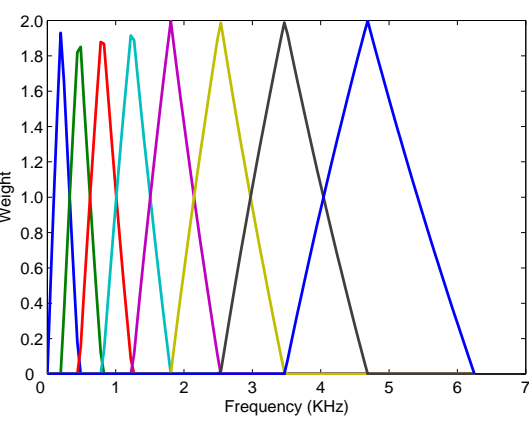

(b)

Fig. 1. (a) Relationship between Hertz and Mels scales. (b) Mels frequency filterbank. The vertical axis represents the weights of filter coefficients.

$\mathrm{dB}$ above the perceptual hearing threshold, is defined as 1000 Mels. Therefore, we can use the following formula to approximate the Mels for a given frequency $f$ in Hertz,

$$
\operatorname{Mel}(f)=2595 \log _{10}\left(1+\frac{f}{700}\right) .
$$

One approach to simulate the subjective spectrum is to use a filter bank, spaced uniformly on the Mel scale as shown in Fig. 1-b. This filter bank is applied to the spectrum of the speech signal to get a Mel-spectrum. The Mel-spectrum when transformed back to time domain using the Discrete Cosine Transform (DCT) gives us the MFCC coefficients. Therefore, if we denote the Mel power spectrum coefficients by $S_{k}$ (where $k$ is the index of the Mel-spaced filters and $k=1,2, \ldots, K)$ then the MFCC coefficients $\left(c_{n}\right)$ are calculated as,

$$
c_{n}=\sum_{k=1}^{K}\left(\log S_{k}\right) \cos \left[n\left(k-\frac{1}{2}\right) \frac{\pi}{K}\right] ; n=1,2 \ldots, L .
$$

We represent MFCC coefficients calculated in Eqn. 2 as an MFCC feature vector,

$$
\mathbf{c}=\left[\begin{array}{lllll}
c_{1} & c_{2} & c_{3} & \ldots & c_{L}
\end{array}\right]^{T}
$$

In this paper, we consider second order statistical modeling of the speech, assuming a wide sense stationary process (WSS) as proposed in [23]. Let $j$ be the index of a class and let there be a total of $N$ classes so that $j=1,2, \ldots, N$. Suppose there are $b$ training utterances available per class. Each utterance from class $j$ is framed using a $20 \mathrm{~ms}$ window so that the assumption of stationarity is true for each frame. Suppose after the framing of all training utterances from class $j$ we end up with $h_{j}$ number of frames. We now calculate 30 MFCC features for each of the $h_{j}$ frames using 30 filter banks (i.e $L=K=30$ in Eqn. 3) and place them in the columns of a matrix $\mathbf{C}_{j}$, so that the matrix is of order $30 \times h_{j}$ :

$$
\mathbf{C}_{\mathbf{j}}=\left[\mathbf{c}_{1}\left|\mathbf{c}_{2}\right| \ldots \mathbf{c}_{h_{j}}\right]
$$




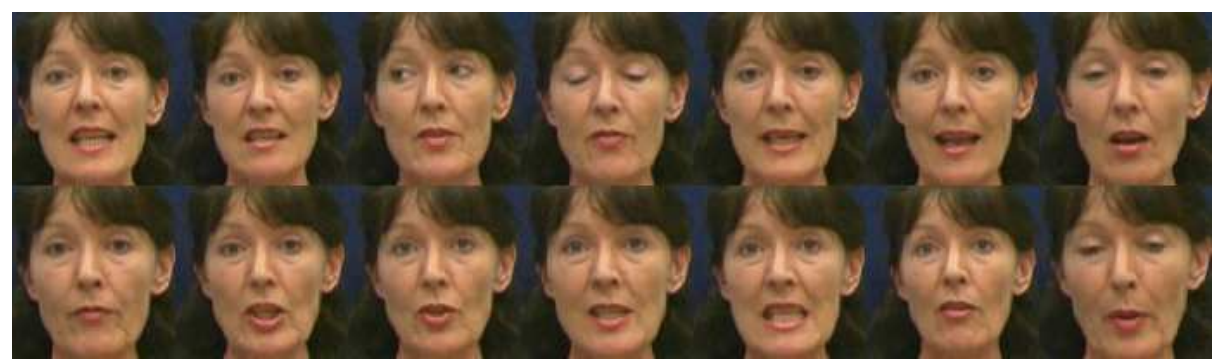

Fig. 2. A sample video sequence illustrates changes in facial expressions while speaking.

Now we develop second order statistical model $\mathbf{U}_{j}$ for class $j$ as,

$$
\begin{gathered}
\hat{\mathbf{c}}=\frac{1}{h_{j}} \sum_{i=1}^{h_{j}} \mathbf{c}_{i}, \\
\mathbf{U}_{\mathbf{j}}=\frac{1}{h_{j}} \sum_{i=1}^{h_{j}}\left(\mathbf{c}_{i}-\hat{\mathbf{c}}\right)\left(\mathbf{c}_{i}-\hat{\mathbf{c}}\right)^{T} .
\end{gathered}
$$

We use the same procedure to develop the above covariance model $\mathbf{U}_{j}$ for all classes i.e $j=1,2, \ldots, N$. Similarly, for a test utterance $z$, we derive a covariance matrix Z Z. Once we have developed the second-order statistical model, we apply an arithmetic-harmonic sphericity measure [23] as the distance metric between all class models $\mathbf{U}_{\mathbf{j}} ; j=1,2, \ldots, N$ and $\mathbf{Z}$, thus

$$
D_{s}\left(\mathbf{U}_{\mathbf{j}}, \mathbf{Z}\right)=\log \left[\frac{\operatorname{tr}\left(\mathbf{U}_{\mathbf{j}} \mathbf{Z}^{-1}\right) \operatorname{tr}\left(\mathbf{Z U}_{\mathbf{j}}{ }^{-1}\right)}{L^{2}}\right] ; j=1,2, \ldots, N
$$

where $L$ is the dimension of the feature vector and $\operatorname{tr}(\mathbf{A})$ is the trace of matrix A. The test pattern $z$ is assigned to the class having the minimum distance measure $D_{s}$.

\section{Video-based Face Recognition}

In any face recognition system, the first step is to detect the face in an image. We used the Haar-like feature based face detection algorithm [24] for detecting the face in a frame. Video-based face recognition was then performed by matching local features of the face as opposed to holistic faces. Local features were chosen because of their robustness to facial expressions. Fig. 2 shows sample faces detected in a video sequence where the subject's facial expressions change significantly with speech.

We used the Scale Invariant Feature Transform (SIFT) [25] for extracting local features from the face. An advantage of using SIFT is that it does not require the prior identification of landmarks on the face. SIFTs are extracted at arbitrary keypoints on the face and do not conform to any specific landmarks (e.g. nose tip). Keypoints are automatically detected as the scale space extrema 
in the Difference-of-Gaussian function convolved with the image. Additionally, the image patch surrounding a keypoint must exhibit high contrast, good localization along an edge and principal curvature ratio of above a threshold.

A histogram is formed from the image patch, surrounding each keypoint, using its gradient orientations weighted by their magnitudes and by a circular Gaussian window. One or more dominant gradient directions are used to align the histogram for rotation invariance. The image patch is divided into $4 \times 4$ sample regions and from each region, an eight bin histogram is extracted resulting in a 128 dimensional feature vector i.e. SIFT. For illumination robustness, the vector is normalized to unity, thresholded to a ceiling of 0.2 and finally renormalized to unit length.

Each detected face in a video frame was rescaled to $150 \times 150$ and histogram equalized before extracting SIFTs. For the training videos, SIFT were extracted offline and stored in the database. During online recognition, faces were automatically detected [24] in the test video sequence, rescaled to $150 \times 150$ and histogram equalized in a similar way before extracting SIFTs. A SIFT vector $v_{q}$ from the test (or query) video was matched with that of a training (or gallery) video using the equation,

$$
e=\cos ^{-1}\left(\mathbf{v}_{q}\left(\mathbf{v}_{g}\right)^{T}\right),
$$

where $\mathbf{v}_{g}$ corresponds to a SIFT vector from a training (or gallery) video. Eqn. 8 was used to match all SIFT features from the query frame to a training frame. Pairs of SIFTs which had the minimum error $e$ were considered matches. If a SIFT from a gallery frame was the best match to more than one SIFT from the same query frame, only the one with the minimum value of $e$ was considered. This removed many errors by allowing only one-to-one matches. Other erratic matches were removed by imposing a distant constraint to match SIFTs from only nearby regions of the two images.

Due to these constraints, different image pairs end up with a different number of matches which serves as an additional measure of similarity between the two faces. The overall similarity between two frames was determined by normalizing the average error $\bar{e}$ between their matching pairs of SIFTs and the total number of matches $m$ on a scale of 0 to 1 and then fusing them using a weighted sum rule.

$$
\begin{gathered}
\bar{e}^{\prime}=\frac{\bar{e}-\min (\bar{e})}{\max (\bar{e}-\min (\bar{e}))}, \\
m^{\prime}=\frac{m-\min (m)}{\max (m-\min (m))}, \\
s=\frac{1}{2}\left(w_{e} \bar{e}^{\prime}+w_{m}\left(1-m^{\prime}\right)\right),
\end{gathered}
$$

where $w_{e}$ and $w_{m}$ are the corresponding weights given to the normalized average error $\bar{e}^{\prime}$ and the normalized number of matches $m^{\prime}$. Note that $\bar{e}^{\prime}$ has a negative 


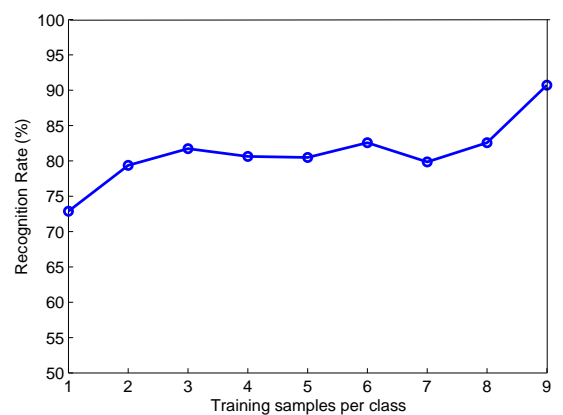

Fig. 3. Speaker recognition performance ( $y$-axis) with varying number of training samples ( $x$-axis). Validation was performed on the remaining $10-x$ samples.

and $m^{\prime}$ has a positive polarity which is why $m^{\prime}$ is subtracted from 1 . The polarity of the final similarity measure $s$ is also negative i.e. lower values mean higher similarity. The final similarity score of a test sequence to a training set was calculated as the average of the minimum $s$ measures per frame of the training set. Details are given in the next section.

\section{Results}

We validate the performance of the proposed audio-visual biometric system on the VidTIMIT $[19,18]$ database. The database consists of 10 audio-video samples each from 43 different subjects. The audio files contain ten, text-independent utterances from each subject while the corresponding video file is a sequence of $512 \times 384$ JPEG images. Each utterance is available as a 16 -bit $32-\mathrm{kHz}$ mono PCM file. Even though the focus of this paper is user verification, we also report the recognition (identification) performance so that the results can be compared to other techniques in the literature [18].

We performed extensive experiments with various combinations of training and testing data. For the speaker recognition module, the rank one recognition rate for varying number of training samples is shown in Fig. 3. The remaining samples were used for validating the system. It is important to emphasize here that the during validation, only one sample per individual was used at a time for his/her verification or identification.

Fig. 4-a shows the rank recognition curves for four combinations of training and validation samples. A rank $x$ recognition rate means the rate at which the correct identity is among the top $x$ identities. We achieved a rank one recognition rate of $79.4 \%$ when the system was trained on two utterances per speaker i.e. T2V8 which stands for 2 training samples and 8 validation samples. The ROC (Receiver Operating Characteristics) curves for four combinations of training and validation samples are shown in Fig. 4-b. Note that increasing the number of training samples beyond two has no significant effect on the systems performance except in the case of T9V1 where the recognition rate jumps to $90.7 \%$ (see Fig. $3)$. However, this is most likely due to the weak evaluation of the system on only one sample per individual. Except for T9V1, the response of the system is 


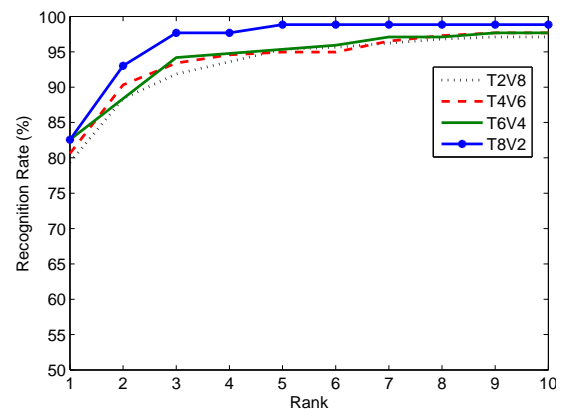

(a)

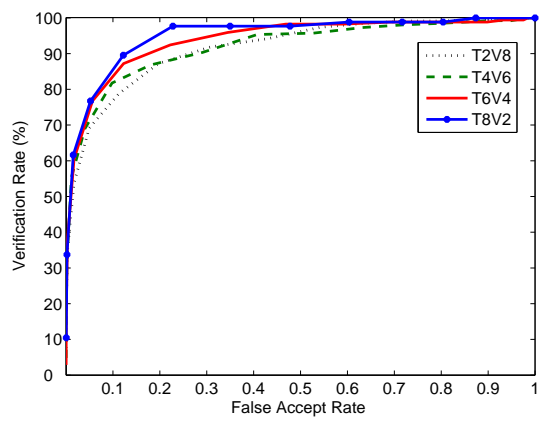

(b)

Fig. 4. (a) Recognition performance for four combinations of test and validation samples. (b) ROC curves for four combinations of test and validation samples.

quite uniform achieving a rank one recognition rate of about $80 \%$. Therefore, for the video-based face recognition module and the overall audio-visual recognition system, we use the minimum number of reliable training samples of two per individual. In other words, the rest of the experiments are performed using the T2V8 setup. The equal error rate of the speech independent speaker verification module was $5.4 \%$ and its verification rate at 0.01 FAR was $85.5 \%$.

Evaluation of the temporal face recognition module was performed using two video sequences for training and eight for testing yielding an identification rate of $93.9 \%$. Again, a single test sequence per person was considered for a single recognition trial. Moreover, alternate frames were matched given that there is high correlation between consecutive frames. The equal error rate of the video classifier was $1.9 \%$ and its verification rate at $0.01 \mathrm{FAR}$ was $97.3 \%$. The rank profile and the ROC curves are shown in Fig. 5-a and 5-b respectively. Fusion of the two modalities was preformed at the score level using a weighted sum rule. Note that this a heterogeneous fusion scenario where the similarity measures for multiple frames in a video sequence must be mapped to similarity measures of only one utterance. The problem grows more complex considering that the similarity matrix for each video frame is a collection of distance measures of the multiple SIFT features of the test frame with all the features in the gallery. This multiform fusion is tackled by opting for the class-based best match in the first step. This best match approach gives us the training frame from each class which yields minimum distance from a given test frame. In the second step, the problem is further simplified by taking an average of all the similarity scores corresponding to all the frames in one video sequence giving us a single similarity score per test video which is in harmony with the similarity score from the speaker recognition module and can be fused accordingly.

For a given test sequence, the similarity scores from speech and face are normalized separately on the scale of zero to one so that the best match (out of the 43 identities in the gallery) has a zero score and the worst has a score of one. The normalized scores are then fused using a weighted-sum rule. Without any prior knowledge of the individual classifiers, it is customary to give them equal 


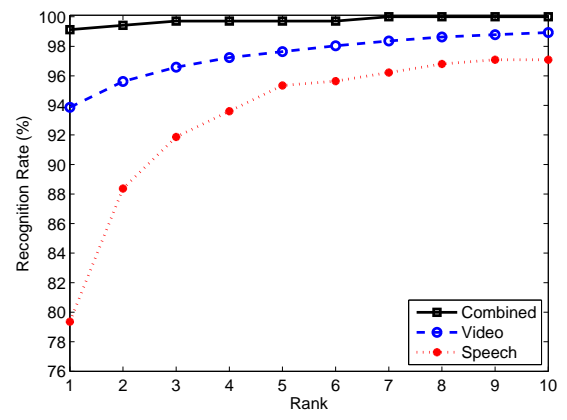

(a)

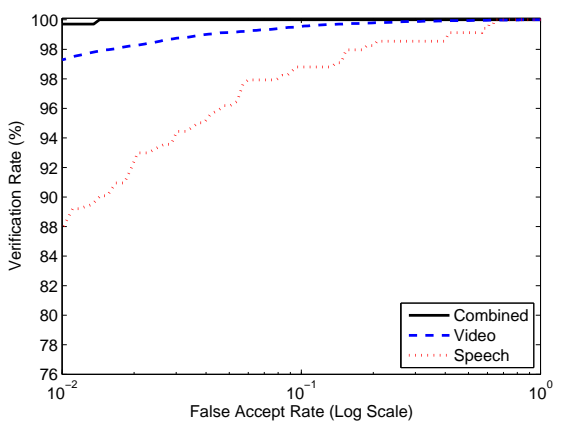

(b)

Fig. 5. (a) Individual and combined recognition performance of the audio-visual system. (b) ROC curves for the individual and combined modalities.

weights. The proposed audio-visual framework achieved a rank one recognition rate of $99.13 \%$ with equal weights. The equal error rate dropped to $0.6 \%$ after fusion and the verification rate at $0.01 \mathrm{FAR}$ increased to $99.7 \%$. The rank recognition and ROC curves for speech, video and their combined performance are shown in Fig. 5-a and Fig. 5-b respectively. A summary of our results is given in Table 1.

Table 1. Summary of results.

\begin{tabular}{|l|c|c|c|}
\hline & Identification Rate & Equal Error Rate & Verification Rate at 0.01 FAR \\
\hline Speech & $79.4 \%$ & $5.4 \%$ & $85.3 \%$ \\
\hline Video Face & $93.9 \%$ & $1.8 \%$ & $97.3 \%$ \\
\hline Combined & $99.1 \%$ & $0.6 \%$ & $99.7 \%$ \\
\hline
\end{tabular}

\section{Conclusion}

We presented a fully automatic (including the offline training phase) user authentication system which combines speech and face biometrics in a video sequence. Face and speech are natural choices from physiological and behavioral biometrics as they are non-intrusive and can be acquired simultaneously using commercial video cameras. The combination of these two biometrics not only improves the accuracy of the verification system but also increases its robustness to impersonation attacks. Experiments were performed on the VidTIMIT database and an equal error rate of $0.6 \%$ and an identification rate of $99.13 \%$ was achieved.

\section{Acknowledgments}

This research is sponsored by UWA Research Grant 2007 and ARC Discovery Grant DP0881813. 


\section{References}

1. Furui, S.: An Overview of Speaker Recognition Technology. In: ESCA Workshop on Automatic Speaker Recognition, Identification and Verification. (1994)

2. Pawlewski, M., Jones, J.: Speaker Verification: Part 1. Biometric Technology Today 14(6) (2006) 9-11

3. Reynolds, D.: A Gaussian Mixture Modeling Approach to Text-independent Speaker Identification. PhD Thesis, Georgia Institute of Technology (1992)

4. McLachlan, G.: Mixture Models. Volume Wright, J. and Yang, A. and Ganesh, A. and Sastri, S, S. and Ma, Y. Marcel Dekker (1988)

5. Tishby, N.: On the Application of Mixture AR Hidden Markov Models to Textindependent Speaker Recognition. IEEE Trans. on Signal Proc. 39 (1991) 563-570

6. Poritz, A.: Linear Predictive Hidden Markov Models and the Speech Signal. Proceedings of IEEE ICASSP (1982) 1291-1294

7. Rosenberg, A.: Sub-word Talker Verification using Hidden Markov Models. Proceeding of IEEE ICASSP (1990) 269-272

8. Levinson, D.: A Perspective on Speech Recognition. Communication Magazine, IEEE 28 (1990)

9. Kohata, M.: Interpolation of LSP Coefficients using Recurrent Neural Networks. Electronics Letters 32 (1996)

10. Zhao, W., Chellappa, R., Phillips, P.J., Rosenfeld, A.: Face Recognition: A Literature Survey. ACM Computing Survey 35(4) (2003) 399-458

11. Turk, M., Pentland, A.: Eigenfaces for Recognition. Journal of Cognitive Neuroscience 3 (1991) 71-86

12. Belhumeur, P., Hespanha, J., Kriegman, D.: Eigenfaces vs. Fisherfaces: Recognition Using Class Specific Linear Projection. IEEE Trans. on PAMI 19 (1997) 711-720

13. Lee, K., Ho, J., Yang, M., Kriegman, D.: Visual Tracking and Recognition Using Probabilistic Appearance Manifolds. CVIU 99(3) (2005) 303-331

14. Liu, L., Wang, Y., Tan, T.: Online Appearance Model Learning for Video-Based Face Recognition. In: CVPR. (2007) 1-7

15. Lee, K., Kriegman, D.: Online Learning of Probabilistic Appearance Manifolds for Video-based Recognition and Tracking. In: CVPR. Volume 1. (2005) 852-859

16. Li, Y., Gong, S., Liddell, H.: Constructing Facial Identity Surfaces in a Nonlinear Discriminating Space. In: CVPR. Volume 2. (2001) 258-263

17. Sivic, J., Everingham, M., Zisserman, A.: Person Spotting: Video Shot Retrieval for Face Sets. In: CIVR. (2005)

18. Sanderson, C., Paliwal, K.: Identity Verification Using Speech and Face Information. Digital Signal Processing 14(5) (2004) 449-480

19. Sanderson, C.: Biometric Person Recognition: Face, Speech and Fusion. VDMVerlag (2008)

20. Moore, B.: Information Extraction and Perceptual Grouping in the Auditory System. Human and Machine Perception: Information Fusion (1997)

21. Haung, X., Acero, A., Hon, H.: Spoken Language Processing: A Guide to Theory, Algorithm and System Development. Prentice Hall PTR, New Jersey (2001)

22. Moore, B.: Frequency Analysis and Masking. Academic Press, USA (1995)

23. Bimbot, F., Magrin-Chagnolleau, I., Mathan, L.: Second-order Statistical Measures for Text-independent Speaker Identification. Speech Communication 17 (1995) 177-192

24. Viola, P., Jones, M.: Robust Real-Time Face Detection. International Journal of Computer Vision 57(2) (2004) 137-154

25. Lowe, D.: Distinctive Image Features from Scale-invariant Key Points. International Journal of Computer Vision 60(2) (2004) 91-110 